\title{
Evidence of a New Form of Self- Organization in DBD Plasmas: The Quincunx Structure
}

\author{
B. Bernecker ${ }^{1}$, T. Callegari ${ }^{1}$, J.P. Boeuf ${ }^{1,2, *}$ \\ ${ }^{1}$ Université de Toulouse; UPS, INPT; LAPLACE (Laboratoire Plasma et Conversion d'Energie); 118 \\ route de Narbonne, F-31062 Toulouse cedex 9, France. \\ ${ }^{2}$ CNRS; LAPLACE; F-31062 Toulouse, France. \\ $\ddagger$ corresponding author; email : jpb@laplace.univ-tlse.fr
}

\begin{abstract}
Self-organized, stable or dynamic, filamentary structures are known to form in dielectric barrier discharges (DBDs) operating in a glow regime. The common "classical" understanding of stable filamentary structures in DBDs is that a glow discharge filament forms at each half-cycle of the sinusoidal applied voltage at the same spatial location along the dielectric surface. The "memory" charges deposited by the previous filament on the dielectric surfaces are responsible for the ignition of a new discharge at the same location. On the other hand, the formation of a filamentary discharge at a given location inhibits discharge development in its vicinity because of charge spreading along the dielectric surface leading to a decrease of the gap voltage in this region. In this paper we show, using experiments and models that another self-organized filamentary regime is possible and coexists with the "classical" regime. In this regime, never reported before, discharge filaments at successive half cycles do not occur at the same location but are shifted by half a spatial period. We call this regime the "quincunx" regime. We also show that the mechanisms of this regime may be responsible for some dynamical aspects of self-organization in DBDs.
\end{abstract}

\section{Introduction}

Dielectric Barrier Discharges (DBDs) are electric discharges between electrodes covered with a dielectric layer and excited by sinusoidal or pulsed voltages. The dielectric layers play the role of capacitance and limit the discharge current. DBDs are the easiest way to generate and control non equilibrium plasmas at elevated pressures and this explains the numerous applications of these discharges ${ }^{1}$, from ozonizers to polymer or textile surface treatment applications, and to UV excimer sources or plasma display panels.

DBDs are also a very interesting and fascinating tool to study self-organized patterns in spatially extended nonlinear dissipative systems ${ }^{3}$. When operating in a simple parallel plate and glow discharge regime the plasma of DBDs is generally not uniform and consists of many filaments perpendicular to the electrodes, sometimes arranged in organized structures, and that can be observed through transparent electrodes. These filaments, termed as "dissipative solitons" (DS) in Ref. [3], exhibit particle-like behaviour. They can form hexagonal patterns representing a crystal-like state as well as liquid- and gas-like patterns, but isolated stationary or travelling DSs interacting through scattering, generation and annihilation phenomena are also observed $^{3}$. Some of the properties of the self-organized filaments are characteristic of reaction-diffusion or Turing systems and attempts have been made to develop semiphenomenological models based on reaction-diffusion equations able to mimic their behaviour ${ }^{4,5}$ (this has however been done mainly for discharges with ohmic and not capacitive layers). In these approaches the variables that are solutions of the reaction-diffusion system may be the local current density and voltage across the gas gap ${ }^{4}$ and the axial behaviour of the discharges is represented only by a typical 
non-linear, s-shaped, current-voltage characteristics. This approach is very interesting and useful to find the necessary "ingredients" in a reaction-diffusion system that allow to mimic the behaviour of the many-body system formed by the filaments and to study the properties of the resulting non-linear system. More work is however needed to improve the justification of these ingredients in term of basic discharge physics. Another approach, based on the basic physics of a glow discharge represented by a simple fluid model (drift-diffusion Poisson) has been able to reproduce and explain the formation of glow-discharge filamentary structures in DBDs ${ }^{6-8}$.

In this paper we report experimental and modelling results that show evidence of the existence of a filamentary regime of DBDs that is not consistent with the common understanding that DBD filaments in a stationary pattern take place at the same location at each half cycle. This new regime, called the "quincunx" regime because filaments of a given half cycle take place in between filaments of the previous cycle, is observed in quasi 1D DBD arrangements and its existence is predicted and can be explained by fluid models. The existence of this stationary regime is very interesting because it appears that the mechanisms responsible for its existence may also be responsible for some dynamical aspects of filamentary discharges that are not clearly understood.

\section{Experimental arrangement, fluid model, and conditions of a DBD glow regime}

Patterns in DBDs can be observed in quasi two-dimensional (2D) or one-dimensional (1D) structures. In the first case, the discharges take place for example between two parallel disk shaped electrodes covered with a dielectric layer ${ }^{8,9}$. If one of the electrode is transparent (Indium Tin Oxide or fine metallic mesh) a $2 \mathrm{D}$ pattern can be observed through the electrode (the discharge axis is perpendicular to the observation plane). Quasi 1D structures can be formed for example between two facing line electrodes deposited on thin dielectric layers. The width of the electrodes must be on the order of the filament diameter so that only one filament can form at a given location along the electrode. A linear structure of filaments can therefore develop along the line electrodes. Such an arrangement has be used in the experiments of Walhout et al. ${ }^{10}$. The structures than can be observed in this geometry are much less complex than in the $2 \mathrm{D}$ arrangement, but the advantage of the $1 \mathrm{D}$ structure is that it allows to observe the discharges both along the electrodes (where the structures form) and across the electrodes (so the details of discharge formation can be viewed with a fast camera). We call $x$ the direction across the electrodes (discharge axis) and $y$ the direction along the electrode. Another advantage of the $1 \mathrm{D}$ arrangement is that the experimental results can be directly compared with results from $2 \mathrm{D}$ discharge models where simulations are performed in the $(x, y)$ plane.

In this paper we use a $1 \mathrm{D}$ experimental arrangement and the experimental results are compared with results from a $2 \mathrm{D}$ fluid model similar to the one used in Refs. [11, 12] to describe plasma display panel DBDs, and in Refs. $[6,7]$ in the context of pattern formations in DBDs. The experimental set-up is shown in Fig. 1. The electrode arrangement of Fig. 1 is placed in a vacuum chamber that can be pumped and filled with a gas mixture. In this paper we show results obtained in neon at pressure around 50 torr, ambient temperature.

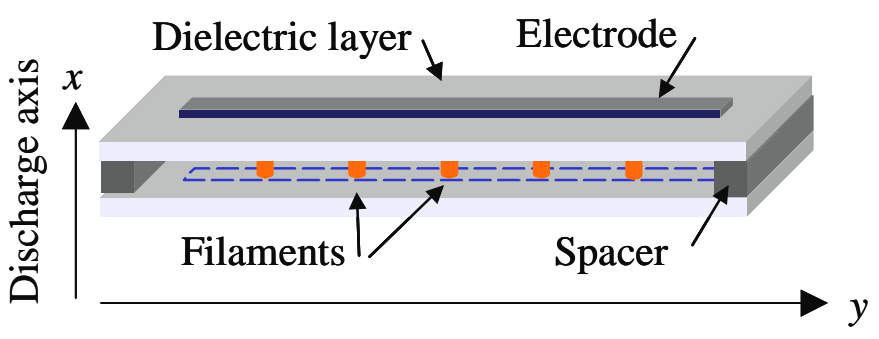

Figure 1: Quasi 1D DBD configuration used in the experiments. The $1 \mathrm{~mm}$ width, $7 \mathrm{~cm}$ length electrodes are deposited on $1 \mathrm{~mm}$ thick epoxy dielectric layers. The gas gap is $3 \mathrm{~mm}$ and 50 torr neon is used in the experiments.

It is very difficult to reproduce, with the model, the same features as in the experiments at the same discharge conditions. This is because the DBD in a filamentary regime is a very non-linear system that can be sensitive to many details (gas impurities, dielectric surface properties) beyond the global discharge parameters (nature of the gas, pressure, voltage and frequency). Small variations in these "details" or in the global discharge parameters may trigger bifurcations from a self-organized stationary regime to another one or to a dynamical regime.

In the modeling part of this work we therefore did not try to reproduce the discharge in the exact conditions of the experiments, but the aim was rather to show that different discharges regimes observed in the experiments can be reproduced and explained by the model under similar (not necessarily identical) conditions. We also wanted to show that the simplest possible discharge model, containing the basic, first order features (drift-diffusion electron and ion transport with direct electron impact ionization depending on the local electric field, secondary electron emission by ion impact on the surfaces, and Poisson's equation) is sufficient to reproduce many features of the selforganization phenomena in DBDs.

The conditions considered in the model results presented in this paper are: DBD in neon, with dielectric thickness and gas gap of $2 \mathrm{~mm}$, electrode length of $2 \mathrm{~cm}$, gas pressure of 50 torr, voltage frequency of $20 \mathrm{kHz}$, secondary electron emission coefficient of neon ions on the surface equal to 0.3 . The relative permittivity of the dielectric is 5 , and symmetry boundary conditions are used at the end of the electrodes in the $y$ direction.

Under the conditions considered in the experiments and in the models the DBDs operate in a transient glow discharge regime, i.e. the electron multiplication in the gap is sufficiently low that discharge breakdown and maintenance is the result of electron multiplication in the volume 
associated with secondary electron emission at the surface (i.e. the regime is far from a streamer regime).

\section{Observation of "classical" and "quincunx" filamentary structures through experiments and models}

Figure 2 shows a typical picture of a periodic filamentary structure in the quasi 1D experimental arrangement of Fig. 1. The maxima of the light emission close to the dielectric surfaces correspond to the negative glow regions of the discharges. Since the picture is integrated in time these maxima are observed on both sides of the gas gap. Each electrode plays in turn the role of a cathode and of an anode, and the maxima in the light emission actually take place alternatively in front of each electrode at successive half cycles of the sinusoidal voltage. The periodic pattern of Fig. 2 therefore corresponds to the "classical" filamentary regime where the discharges form at the same spatial locations along the electrode at each half cycle (in opposite directions, i.e. each electrode is alternatively a cathode and an anode).

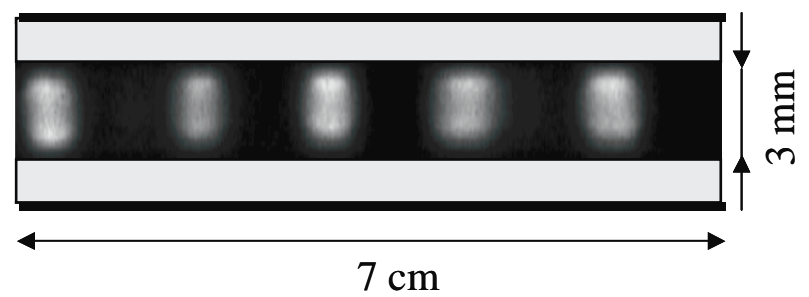

Figure 2: Picture of a "classical" quasi 1D periodic filamentary structure in the electrode configuration of Fig. 1. The discharge is in 50 torr $\mathrm{Ne}$ and the amplitude and frequency of the sinusoidal applied voltage are $450 \mathrm{~V}$ and $10 \mathrm{kHz}$ respectively. The exposure time is 1 cycle $(100 \mu \mathrm{s})$ and the image is integrated over a large number of cycles.
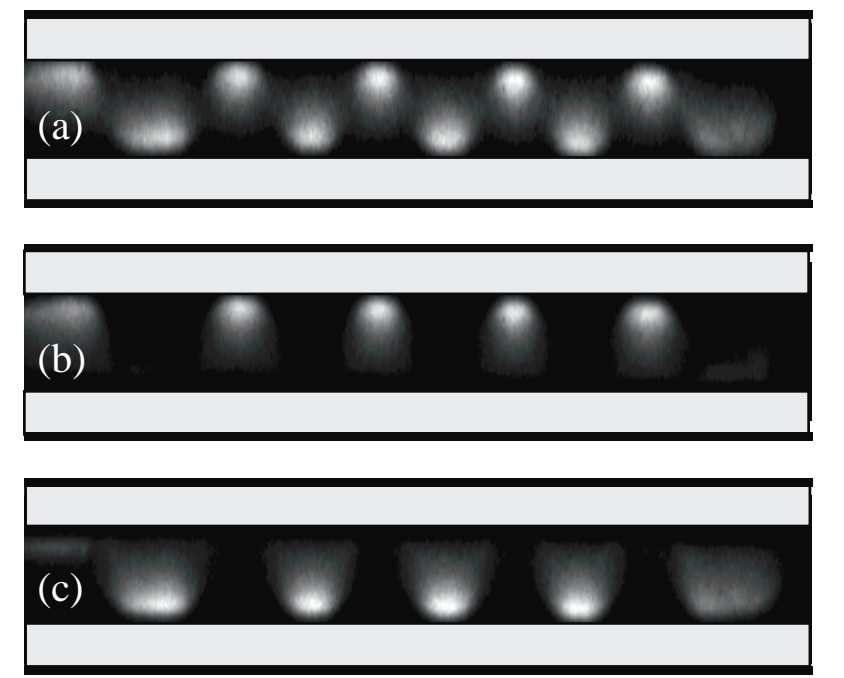

Figure 3: Picture of a filamentary "quincunx" structure in the linear electrode arrangement of Fig. 1, in neon, 50 torr with voltage amplitude and frequency $524 \mathrm{~V}$ and $30 \mathrm{kHz}$ respectively; the picture of (a) is integrated over one cycle of the applied voltage (and accumulated over many cycles), while the pictures of (b) and (c) are taken over two different half cycles and accumulated over many cycles.
Our observations show that the "classical" filamentary regime of Fig. 2 can bifurcate toward a different, unexpected regime, never reported before, where the filaments of successive half cycles do not take place at the same locations along the electrodes, but are shifted from the pattern of the previous half cycle by half a spatial period (see Fig. 3). This filamentary periodic structure, that we will call "quincunx" structure has therefore a spatial period equal to half that of the "classical" structure. The filaments at a given half cycle of the voltage do not form at the same locations as the filament of the previous half cycle, but in between these filaments.

The existence of the quincunx structure is quite unexpected since our understanding of pattern formation in DBDs relies on the fact that filaments form preferentially where memory charges have been deposited during the previous half cycle and inhibit the formation of filaments in their vicinity because the spreading of the charges on the dielectric surface is larger than the size of each filament ${ }^{6-8}$. In term of reaction-diffusion systems, volume ionisation (due to the gap voltage) and surface charge (the voltage due to memory charges deposited by the previous filament adds to the gap voltage) play the role of an activator, while surface charging in a second phase plays the role of an inhibitor since the decrease of the gap voltage in the vicinity of a forming filament due to charge spreading along the dielectric surface limits discharge formation around this filament.

Although this image is correct, it is also possible, under some conditions, that the voltage drop associated with the spreading of the charges along the dielectric layers during filament formation at a given half cycle does not completely quench discharge formation between these filaments but allows the formation of a lower current discharge (e.g. much less luminous "Townsend" discharge). This has been illustrated in the experimental and modelling results of Bernecker et al. ${ }^{13}$.

Although the current density of the Townsend discharges between filaments is much less than the current density of the glow discharge filaments, the duration of these low discharge currents can be much longer and lead to a total charge transferred between filaments on the same order as the charge transferred by the filaments. Moreover the model also shows that, because of the longer duration of the Townsend discharges, the density of remaining ions in the gap at the location of these discharges can be much larger than at the locations of the filamentary discharges. This higher remaining volume charge density between filaments, combined with the fact that the charge transferred by the filaments and by the discharges between filaments are equivalent lead, at the next half cycle, to the triggering of discharges in between the filaments of the previous halfcycle. This qualitative explanation is supported by the $2 \mathrm{D}$ fluid model, which is able to predict the bifurcation from the classical to the quincunx regimes when some discharge parameters are changed. Figure $4 \mathrm{a}$ and $4 \mathrm{~b}$ show the time integrated electron densities and ionisation rate in the quincunx regime as provided by the model. 
$2 \mathrm{~cm}$



(b)

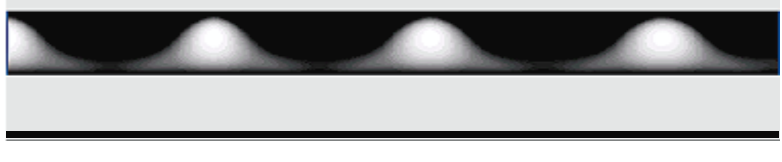

(c)
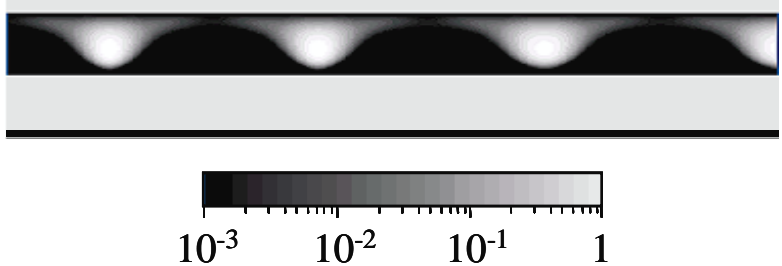

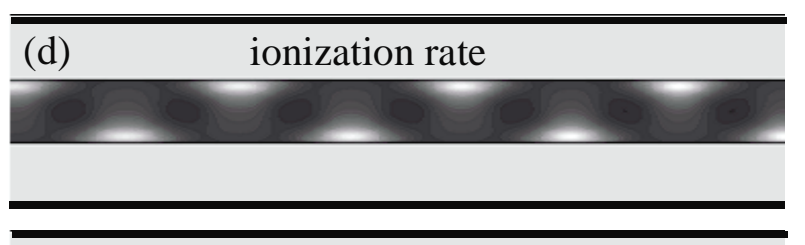

(e)

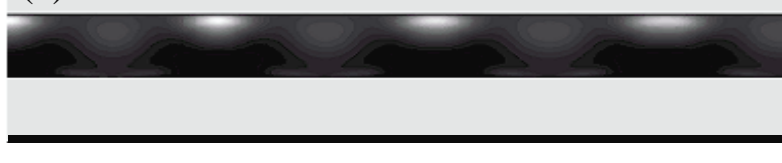

(f)

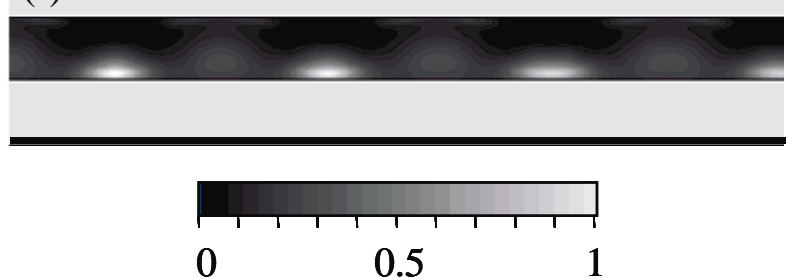

Figure 4: Space distribution of the electron density (a), (b), (c), and ionisation rate (d), (e), (f) from the 2D model in a quincunx filamentary regime neon, 50 torr, $700 \mathrm{~V}$ and $20 \mathrm{kHz}$ voltage amplitude and frequency; (a), (d) the density and ionisation rate are averaged over one cycle, (b), (e) over a half-cycle, (c), (g) over the following half-cycle. The densities are plotted on a 3 decades log scale, with a maximum of $210^{9} \mathrm{~cm}^{-3}$ for (a) and $410^{9} \mathrm{~cm}^{-3}$ for (b), (c). The ionisation rates are on a linear scale with maximum $0.510^{16}$ $\mathrm{cm}^{-3} \mathrm{~s}^{-1}$ for (d), and $10^{16} \mathrm{~cm}^{-3} \mathrm{~s}^{-1}$ for (e) and (f).

Figure 5a shows the time integrated picture of an experimental linear DBD in neon, 300 torr, $880 \mathrm{~V}$ and 50 $\mathrm{kHz}$ voltage amplitude and frequency, $3 \mathrm{~mm}$ gap. On this figure, the discharge seems to correspond to a static quincunx filamentary regime similar to the one described above (Fig. 11). Figures 5b, c, d, e actually show that the discharge is more complex and present some dynamical features that are periodic with a period equal to three times the period of the applied voltage. The picture of Fig. 5b has been taken with a gate of $10 \mu$ s (i.e. one half-cycle) and show that only $1 / 3$ of the filaments expected in a half-cycle quincunx pattern are actually present. Integrating in time over two successive half-cycles gives the picture of Fig. 5c. The filaments of the second half-cycle are shifted by half a spatial period of the quincunx structure of Fig. 5a (the cathode sheath is on the opposite side with respect to the previous filaments). Figures $5 \mathrm{~d}$ and $5 \mathrm{e}$ show that at each half cycle, the new filaments are shifted by a half a spatial period. The structure of Fig. 5 is therefore not static and presents an apparent organized motion of several filaments from right to left. The spatial period of this motion is equal to 3 periods of the applied voltage (integrating over 6 halfcycles would give a picture close to that of Fig. 5a).

The experimental results of Fig. 5 are very interesting since they show that the motion of filaments can be due to mechanisms similar to those responsible for the existence of the quincunx regime (i.e. remaining charges in the discharge volume close to a filament trigger the next discharge on the side of this filament). The fact that the motion appears to be from right to left in Fig. 5 is not clear (broken symmetry) and may be associated with the discharge history and memory charge effects.
In the $1 \mathrm{D}$ electrode configuration of Fig. 5, we were able to capture many other dynamical features (not reported here) of the filaments, such as filament merging and division. Some of these features could be reproduced with the models. As mentioned above, under specific conditions, the filaments of DBDs are not static but appear to be moving along the dielectric surface and interact with each other (merging or division of filaments). The filaments motion can be chaotic in appearance but is sometimes organized. An example of apparent motion of filaments is shown in Fig. 5.

\section{Conclusion}

Evidence of a new form of self-organized filamentary pattern in a quasi 1D DBD in a glow regime, the "quincunx" structure has been shown experimentally and with fluid models of the discharge. In this regime filaments of a given half cycle of the sinusoidal voltage form between filaments of the previous half cycle, in contrast with the "classical" structure of stationary filamentary DBD patterns. In the quincunx regime, long lasting low current (dark) discharges between filaments transfer a similar amount of surface charge than the filamentary discharges but because of a larger remaining volume charge between filaments, the filamentary discharges at the next half cycle are triggered between the previous ones. The existence of this regime results from a competition between volume and surface charges in triggering the new filaments, and may explain some dynamical aspects of DBD filaments. 

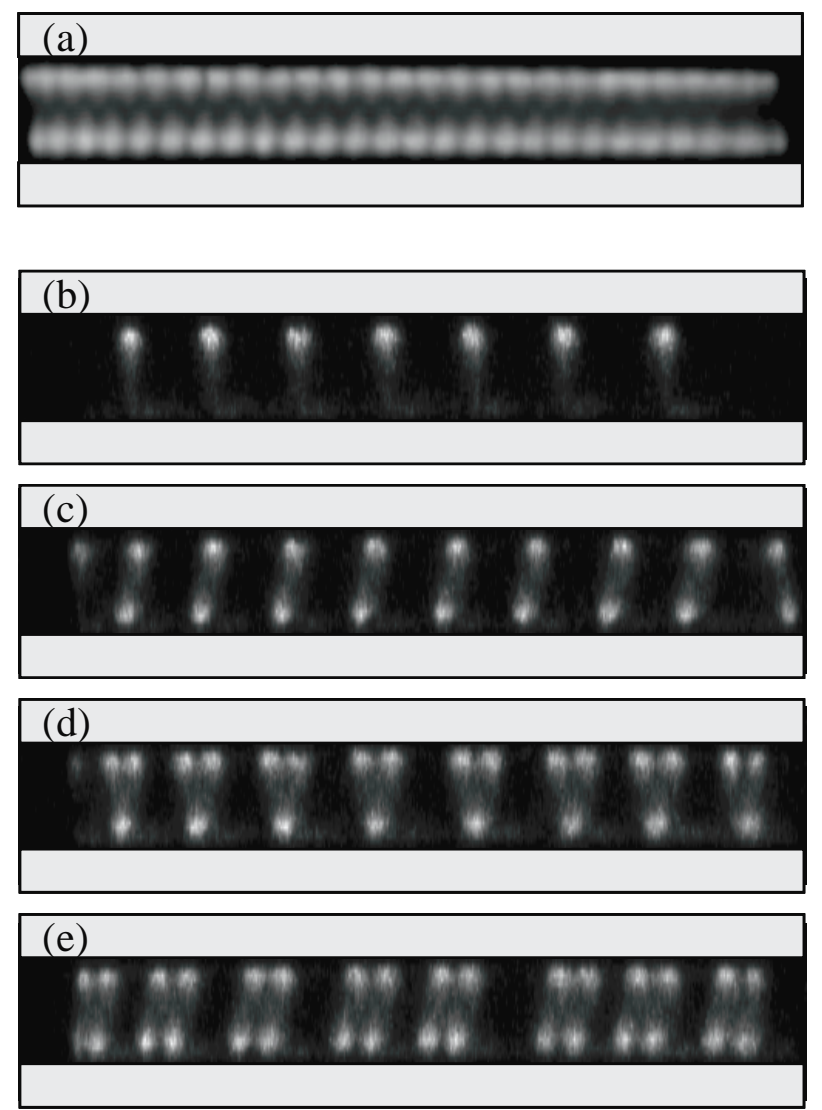

Figure 5 : Multiperiodic filamentary discharge in a quasi 1D DBD experiment in neon, 300 torr, $880 \mathrm{~V}, 50 \mathrm{kHz}, 3 \mathrm{~mm}$ gap, $2 \mathrm{~mm}$ dielectric layers: (a) time integrated picture of the discharge (standard camera); (b), (c), (d), (e), pictures of the discharge taken with an ICCD camera and integrated over gates of 10, 20, 30, and $40 \mu$ s, i.e. 1, 2, 3, 4 half-cycles respectively.

\section{References}

[1] U. Kogelschatz, Plasma Chem. and Plasma Proc. 231 (2003).

[2] J.P. Boeuf, "Plasma display panels- physics, recent developments and key issues", J. Phys. D: Appl. Phys. 36, 6 (2003)

[3] H.G. Purwins, H.U. Bodecker, and S. Aminarashvili, Advances in Physics 59485 (2010)

[4] Sh. Amiranashvili, S. V. Gurevich, and H.G. Purwins, Phys. Rev. E 71, 066404 (2005)

[5] C. P. Schenk, M. Or-Guil, M. Bode and H.-G. Purwins, Phys. Rev. Lett. 78, 3781-3784 (1997)

[6] I. Müller, C. Punset, E. Ammelt, H.-G. Purwins, J.P. Boeuf, IEEE Trans. Plasma Sci. 27, 20 (1999)

[7] I. Brauer, C. Punset, H-G Purwins, J-P Boeuf, J. Appl. Phys. 85, 7569 (1999)

[8] L. Stollenwerk, Sh. Amiranashvili, J.-P. Boeuf and H.-G. Purwins, Phys. Rev. Lett. 96, 255001 (2006).

[9] L.F. Dong, Z.Q. Yin, X.C. Li, and L. Wang, Plasma Sources Sci. Technol. 12380 (2003); L.F. Dong, F.C. Liu, S.H. Liu, Y.F. He, W.L. Fan, Phys. Rev. E 72046215 (2005)

[10] J. Guikema, N. Miller, J. Niehof, M. Klein, and M. Walhout, Phys. Rev. Lett. 85, 3817 (2000)

[11] J.P. Boeuf and L.C. Pitchford, IEEE Trans. Plasma Sci. 2495 (1996)

[12] C. Punset, S. Cany, and J.P. Boeuf, J. Appl. Phys. 86124 (1999)

[13] B. Bernecker , T. Callegari, S. Blanco, R. Fournier and J.P. Boeuf , Eur. Phys. J. Appl. Phys. 4722808 (2009) 